\section{Writing Nano-Scale Patterns on Insulators Using Variable Pressure Electron-Beam Lithography}

\author{
Floyd Miller and David Frey*
}

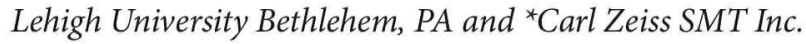
fcm1@Lehigh.edu,frey@smt.zeiss.com

\section{Introduction}

Electron-beam lithography offers very high resolution patterns without the need for masks. Systems based on electron microscopes offer entry into electron-beam lithography at reasonable cost. Such systems are becoming popular in research environments due to their versatility and reasonable cost.

While these systems work well they do have problems writing on insulating substrates. The insulating substrate causes charge to build up from the incident electron-beam. As the charge builds up on the surface of the insulator, the incident beam is distorted and moved in $\mathrm{x}$ and $\mathrm{y}$. The result is a lack of positioning accuracy on the patterns as well as pattern distortion.

To overcome the problem of substrate charging, lithographers resort to placing a layer of gold or other metal on top of the resist. The resist is exposed through the metal and the metal removed before developing the resist. A similar technique is to use a conductive polymer on top of or under the resist to dissipate charge (1). Both of these techniques add complexity to the process as well as increasing the potential of contaminating the substrate.

In the current study, the authors wanted to examine the use of a variable pressure SEM to write patterns without having to coat the samples with metal or other conductive material. This technique would potentially simplify writing on insulators while maintaining high pattern resolution.

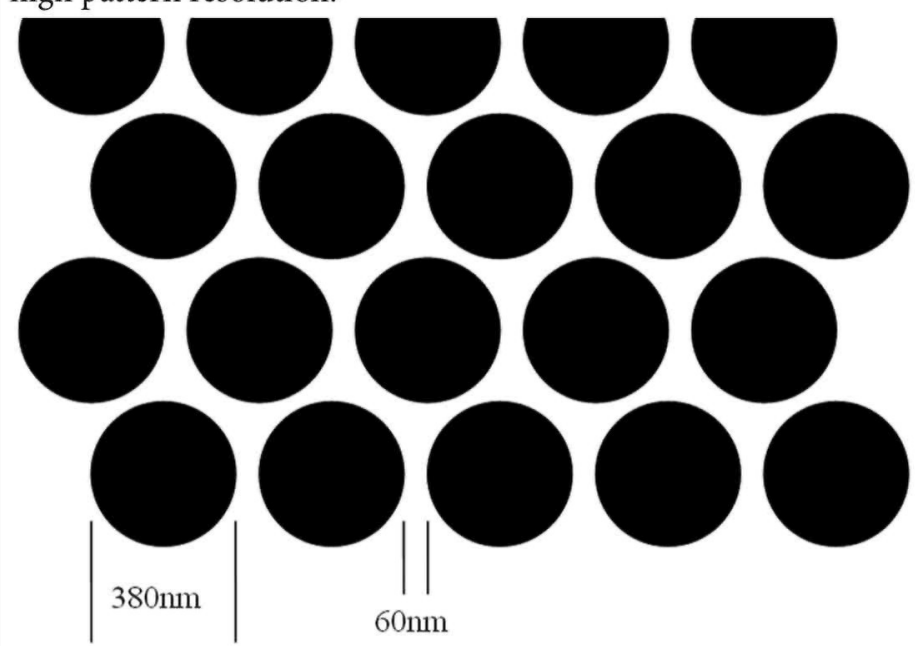

Figure 1. Layout of the pattern used to expose the three samples.

\section{Method}

Three samples were exposed using the pattern in figure 1 . The area inside of the dark circles was written by the beam. All three samples had the same thickness of PMMA photo resist (about 2,000 $\AA$ ). The same SEM was used for each exposure and all were exposed at 30,000 volts. Samples were exposed using either standard high vacuum mode or using variable pressure mode. For variable pressure mode the ambient was nitrogen and the pressure was $30 \mathrm{~Pa}$. All exposures received the same electron dose.

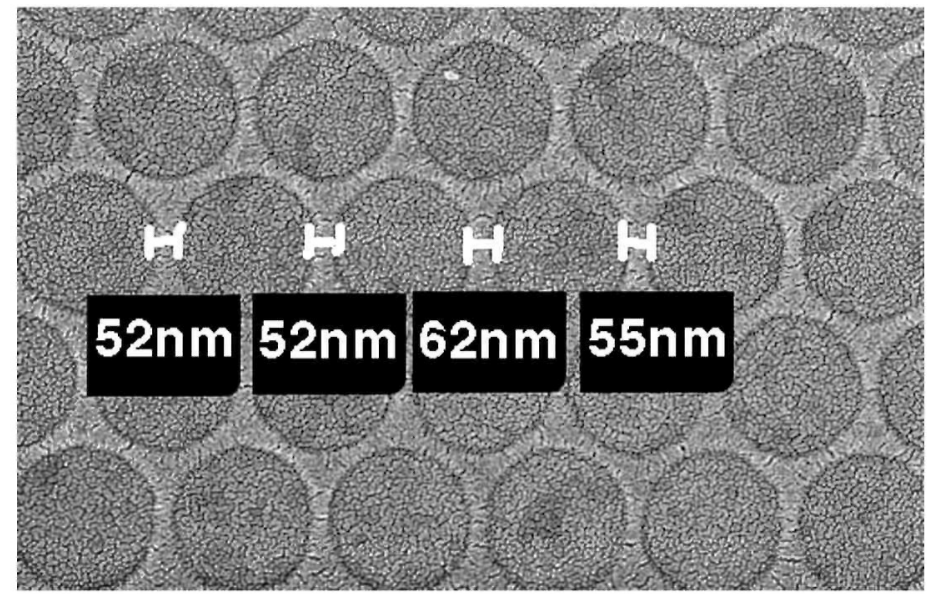

Sample 1. Silicon patterned at low pressure

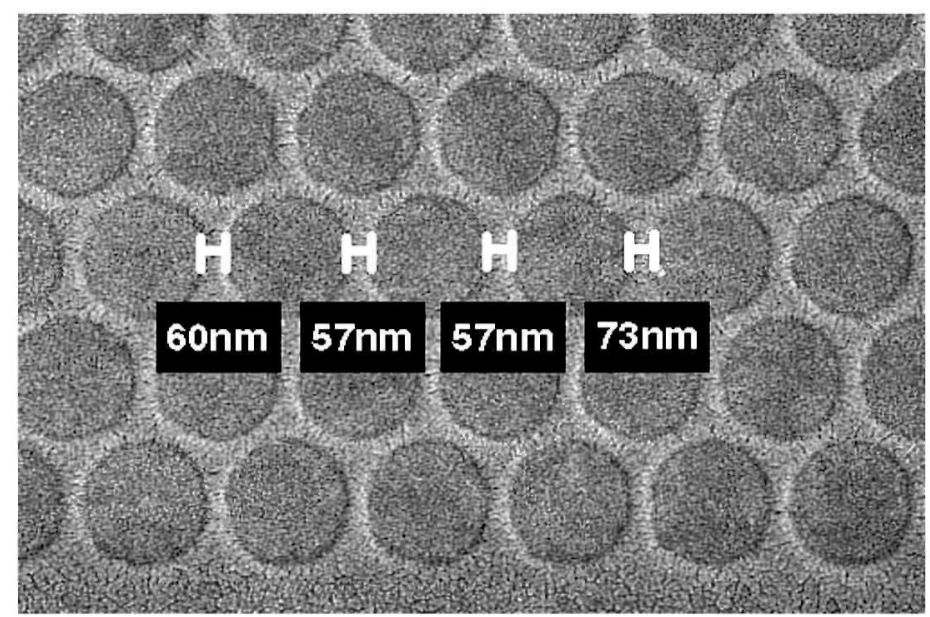

Sample 2. Silicon patterned at $30 \mathrm{~Pa}$.

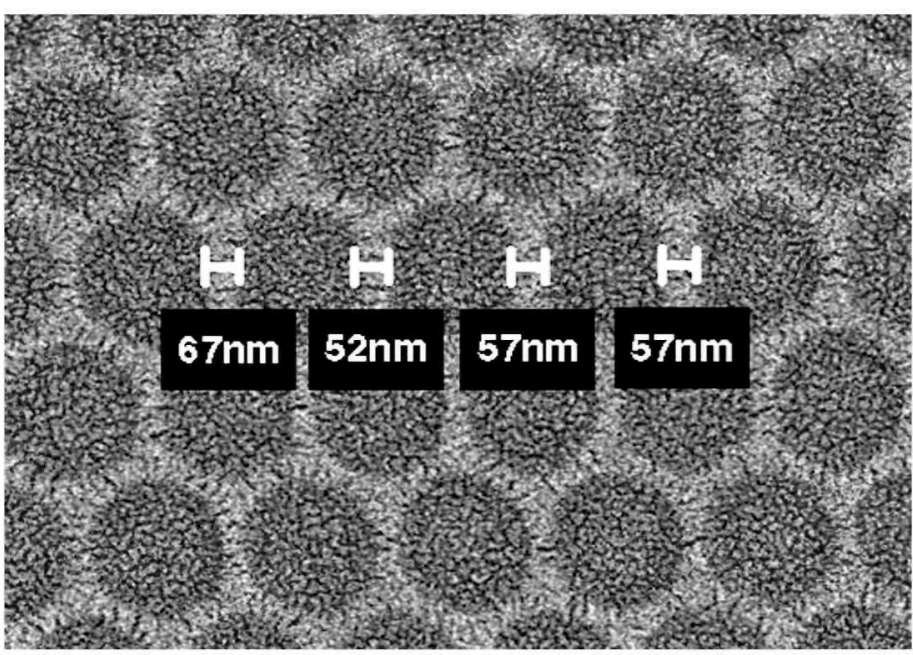

Sample 3. Sapphire patterned at $30 \mathrm{~Pa}$

- Sample 1 was silicon written using high vacuum mode.

- Sample 2 was silicon written using variable pressure mode at $30 \mathrm{~Pa}$.

- Sample 3 was sapphire written using variable pressure mode at $30 \mathrm{~Pa}$.

After exposure, the samples were developed and $50 \AA$ of gold was deposited for SEM imaging. The samples were placed back in the SEM to measure the spacing between the circles. Four measurements were taken of each sample and averaged. 


\section{Results}

Figures 2 through 4 are images of the three samples annotated with the line width measurements. Table 1 lists the measurement results.

\begin{tabular}{|l|c|c|c|}
\hline & $\begin{array}{c}\text { Sample 1 } \\
\text { Silicon, High } \\
\text { Vacuum }\end{array}$ & $\begin{array}{c}\underline{\text { Sample 2 }} \\
\text { Silicon, 30 Pa. }\end{array}$ & $\begin{array}{c}\frac{\text { Sample 3 }}{\text { Sapphire, }} \\
30 \mathrm{~Pa}\end{array}$ \\
\hline Width 1 (nm) & 52 & 59 & 66 \\
\hline Width 2 (nm) & 52 & 57 & 52 \\
\hline Width 3 (nm) & 62 & 57 & 57 \\
\hline Width 4 (nm) & 55 & 73 & 57 \\
\hline Average Width & 55 & 62 & 57 \\
\hline
\end{tabular}

Table 1. Line width measurements of the three samples.

There was a maximum variation of only $7 \mathrm{~nm}$ between the three samples. Given the minimum line width of this system of 15 to $20 \mathrm{~nm}$ it can be seen that the variation within this experiment is less that the width of a single line.

\section{Conclusions}

This work demonstrates that it is possible to write nano-scale patterns on insulators using a variable pressure electron microscope. The use of variable pressure allows writing on insulators without adding any conductive layers during the lithography process. While this work did not explore the ultimate resolution limits of this technique, it did demonstrate that lines of $60 \mathrm{~nm}$ are possible. Further, at the resolution of this experiment, there was no noticeable difference between patterns written in conventional high vacuum mode and those written in variable pressure mode.

\section{Reference}

1. Ed., P. Rai-Choudhury, Handbook of Microlithography, Micromachining, and Micro fabrication, Volume 1, Microlithography, pg 2.7.1

\section{MICROSCOPY FOR CHILDREN}

\section{Caroline Schooley}

New children's microscopy books were introduced at the Microscopy \& Microanalysis meeting in Honolulu this last summer. They deserve your attention, particularly if you work in an SEM lab. These reviews are available at MSA's Project MICRO website, http://microscopy.org/ProjectMICRO/.

Levine, S. Johnstone, L., \& Humphrey, E., 2005 Extreme 3-D: Scary Bugs, Extreme 3-D: Weird Animals, Extreme 3-D: Your Body

8.5 x 11", 48 pp, hardbound, \$ 14.95 each, ISBNs: 1-59223-365-1, 1-59223-364-3, 1-59223-366-x. Silver Dolphin Books, www.silverdolphinbooks.com Shar Levine and Leslie Johnstone have written some of the best children's "how-to" microscopy books. For this new series they are joined by electron microscopist Elaine Humphrey. The result is a series of far better quality than the various "ugly bug" picture books that were published in the '90s. "Scary Bugs" includes insects, spiders, and millipedes, "Weird Animals" are invertebrates, several phyla of vertebrates, and tarantulas, and "Your Body" has body parts plus parasites and bacteria. The micrographs are excellent, the red-green stereo is effective, and the text is well-written and informative. The books are well-made; the binding and 3-D glasses are more durable than most. All three use the same four page explanation of stereo vision and microscopy. Two pages are devoted to each topic; the high magnification images are supported by text and several low magnification views of the organism. Since the low power images aren't 3-D, even those with stereo vision problems will find the books interesting. These three should be in every SEM lab that does tours for visiting classes. Age 8 - adult
Sly, A. \& Kunkel, D., 2005 Mosquito Bite

9x 9", 32 pp, hardback \$ 15.95, ISBN 1-57091-591-1 Charlesbridge Publishing, www.charlesbridge.com, (800)225-3214)

Mosquito Bite isn't "just another ugly bug book"; it presents the mosquito life cycle in the context of childern's backyard play. Dennis Kunkel's electron micrographs are, as usual, excellent. The design of the book will appeal to its intended audience (ages 6-11), but some of the concepts and vocabulary need support from an informed teacher or parent. Current concern about West Nile virus should make it a useful teaching aid.

This following book isn't new, but the very cheap paperback edition is; it's even cheaper on the internet. It's a perfect match with MICRO, and it should be in every classroom that uses MICRO's manual, "Microscopic Explorations".

Kramer, S. \& Kunkel, D. 2001 Hidden Worlds: Looking Through a Scientist's Microscope

11 x 8.5”, 57 pp, paperback, \$5.95, ISBN 0-618-35405-0, Houghton Mifflin Co., www.houghtonmifflinbooks.com

Hidden Worlds is an award-winning (and low cost!) book about the excitement of observation and the beauty of the microworld. The micrographs are excellent, and the types of light and electron microscopes used to produce them are described clearly, without technical jargon. Chapter titles include "Becoming a Scientist", "Working as a Scientist", Seeing Hidden Worlds in Nature", and "How to Become a Scientist". Its dramatic presentation and readable text will capture the imagination of the children who read it. Grades 4-8.

\section{SERPETRENIX PHINE/FAX $(239) 945-6763$ \\ Introducing the MaxView Plus Digital Camera Attachment System}

The MaxView Plus kit includes adapters to fit microscopes \& optical devices with any of the following attachments; C-Mount, C/S-Mount, T-Mount, 23mm Eyepiece Port, $30 \mathrm{~mm}$ Eyepiece Port, and 1.25" Eyepiece Ports

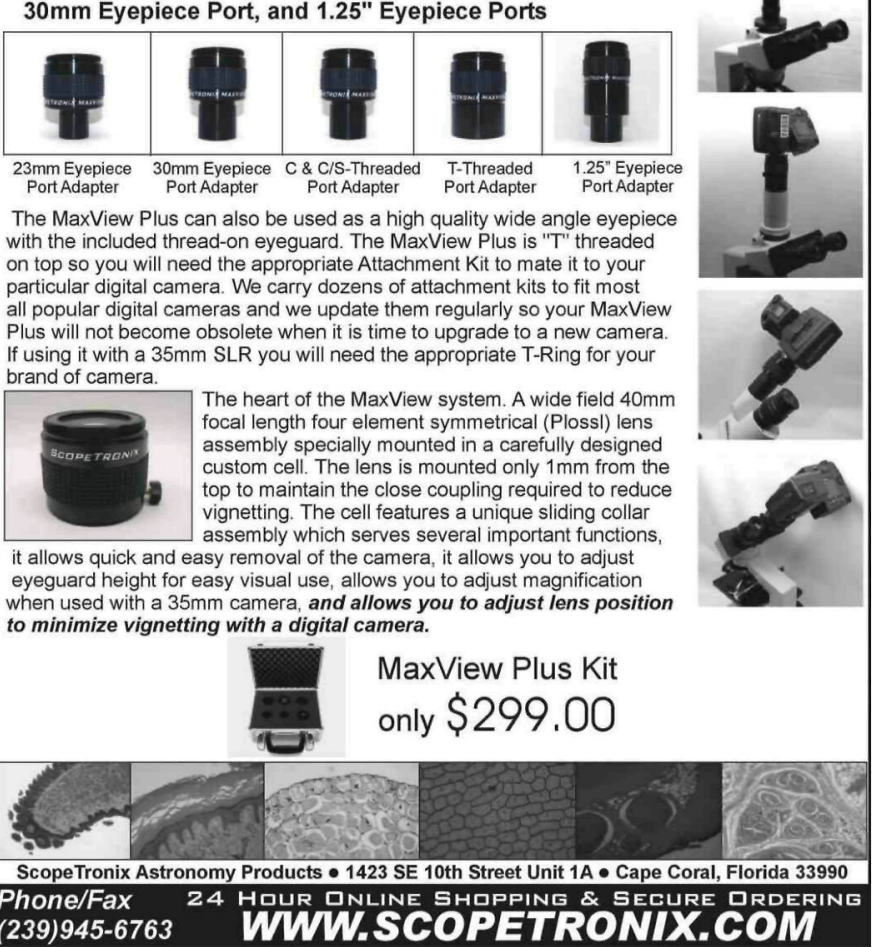

